\title{
Cognitive radio transmission under QoS constraints and interference limitations
}

\author{
Sami Akin ${ }^{1 *}$ and Mustafa Cenk Gursoy²
}

\begin{abstract}
In this article, the performance of cognitive transmission under quality of service (QoS) constraints and interference limitations is studied. Cognitive secondary users are assumed to initially perform sensing over multiple frequency bands (or equivalently channels) to detect the activities of primary users. Subsequently, they perform transmission in a single channel at variable power and rates depending on the channel sensing decisions and the fading environment. A state transition model is constructed to model this cognitive operation. Statistical limitations on the buffer lengths are imposed to take into account the QoS constraints of the cognitive secondary users. Under such QoS constraints and limitations on the interference caused to the primary users, the maximum throughput is identified by finding the effective capacity of the cognitive radio channel. Optimal power allocation strategies are obtained and the optimal channel selection criterion is identified. The intricate interplay between effective capacity, interference and QoS constraints, channel sensing parameters and reliability, fading, and the number of available frequency bands is investigated through numerical results.
\end{abstract}

Keywords: Channel sensing, Cognitive transmission, Effective capacity, Energy detection, Interference constraints, Power adaptation, Quality of service constraints

\section{Introduction}

Recent years have witnessed much interest in cognitive radio systems due to their promise as a technology that enables systems to utilize the available spectrum much more effectively. This interest has resulted in a spur of research activity in the area. Asghari and Aissa [1], under constraints on the average interference caused at the licensed user over Rayleigh fading channels, studied two adaptation policies at the secondary user's transmitter in a cognitive radio system one of which is variable power and the other is variable rate and power. They maximized the achievable rates under the above constraints and the bit error rate requirement in MQAM modulation. The authors of [2] derived the fading channel capacity of a secondary user subject to both average and peak receivedpower constraints at the primary receiver. In addition, they obtained optimum power allocation schemes for three different capacity notions, namely, ergodic, outage, and minimum-rate. Ghasemi and Sousa [3] studied the

\footnotetext{
*Correspondence: sami.akin@ikt.uni-hannover.de

1 Institute of Communications Technology, Leibniz Universität Hannover,

30167 Hanover, Germany

Full list of author information is available at the end of the article
}

performance of spectrum-sensing radios under channel fading. They showed that due to uncertainty resulting from fading, local signal processing alone may not be adequate to meet the performance requirements. Therefore, to remedy this uncertainty they also focused on the cooperation among secondary users and the tradeoff between local processing and cooperation in order to maximize the spectrum utilization. Furthermore, the authors of [4] focused on the problem of designing the sensing duration to maximize the achievable throughput for the secondary network under the constraint that the primary users are sufficiently protected. They formulated the sensing-throughput tradeoff problem mathematically, and use energy detection sensing scheme to prove that the formulated problem indeed has one optimal sensing time which yields the highest throughput for the secondary network. Moreover, Quan et al. [5] introduced a novel wideband spectrum sensing technique, called multiband joint detection, which jointly detects the signal energy levels over multiple frequency bands rather than considering one band at a time.

In many wireless systems, it is very important to provide reliable communications while sustaining a certain level

\section{量 Springer}

(c) 2012 Akin and Gursoy; licensee Springer. This is an Open Access article distributed under the terms of the Creative Commons Attribution License (http://creativecommons.org/licenses/by/2.0), which permits unrestricted use, distribution, and reproduction in any medium, provided the original work is properly cited. 
of quality of service (QoS) under time-varying channel conditions. For instance, in wireless multimedia transmissions, stringent delay QoS requirements need to be satisfied in order to provide acceptable performance levels. In cognitive radio systems, challenges in providing QoS assurances increase due to the fact that secondary users should operate under constraints on the interference levels that they inflict on the primary users. For the secondary users, these interference constraints lead to variations in transmit power levels and channel accesses. For instance, intermittent access to the channels due to the activity of primary users make it difficult for the secondary users to satisfy their own QoS limitations.

These considerations have led to studies that investigate the cognitive radio performance under QoS constraints. Musavian and Aissa [6] considered variable-rate, variablepower MQAM modulation employed under delay QoS constraints over spectrum-sharing channels. As a performance metric, they used the effective capacity to characterize the maximum throughput under QoS constraints. They assumed that two users sharing the spectrum with one of them having a primary access to the band. The other, known as secondary user, is constrained by interference limitations imposed by the primary user. Considering two modulation schemes, continuous MQAM and discrete MQAM with restricted constellations, they obtained the effective capacity of the secondary user's link, and derived the optimum power allocation scheme that maximizes the effective capacity in each case. In addition, in [7], they proposed a QoS constrained power and rate allocation scheme for spectrum sharing systems in which the secondary users are allowed to use the spectrum under an interference constraint by which a minimum-rate of transmission is guaranteed to the primary user for a certain percentage of time. Moreover, applying an average interference power constraint which is required to be fulfilled by the secondary user, they obtained the maximum arrival-rate supported by a Rayleigh block-fading channel subject to satisfying a given statistical delay QoS constraint. We note that in these studies on the performance under QoS limitations, channel sensing is not incorporated into the system model. As a result, adaptation of the cognitive transmission according to the presence or absence of the primary users is not considered.

In this article, we study the effective capacity of cognitive radio channels where the cognitive radio detects the activity of primary users in a multiband environment and then performs the data transmission in one of the transmission channels. Both the secondary receiver and the secondary transmitter know the fading coefficients of their own channel, and of the channel between the secondary transmitter and the primary receiver. The cognitive radio has two power allocation policies depending on the activities of the primary users and the sensing decisions. More specifically, the contributions of this article are the following:

1. We consider a scenario in which the cognitive system employs multi-channel sensing and uses one channel for data transmission thereby decreasing the probability of interference to the primary users.

2. We identify a state-transition model for cognitive radio transmission in which we compare the transmission rates with instantaneous channel capacities, and also incorporate the results of channel sensing.

3. We determine the effective capacity of the cognitive channel under limitations on the average interference power experienced by the primary receiver.

4. We identify the optimal criterion to select the transmission channel out of the available channels and obtain the optimal power adaptation policies that maximize the effective capacity.

5. We analyze the interactions between the effective capacity, QoS constraints, channel sensing duration, channel detection threshold, detection, and false alarm probabilities through numerical techniques.

We would like to note that, in $[8,9]$, we have also studied the performance of cognitive radio systems in the presence of QoS constraints. However, there are significant differences in the models and the analysis between this article and $[8,9]$. In $[8,9]$, channel sensing is done only in one channel. In this article, we consider multi-channel sensing and also identify an efficient rule to decide on which channel to use for transmission among multiple channels. In the previous study, channel conditions between the secondary and primary users are assumed to be unknown. Consequently, interference constraints have a simpler structure. Indeed, in [9], the secondary transmitter does not even know its own channel and sends the information at a fixed rate and power. The secondary receiver only has imperfect knowledge obtained through channel estimation. The performance in such a challenging environment can be regarded as lower bounds on what can be attained in practice. In this article, in order to complete the picture, we consider the flip side of the coin and assume that the secondary transmitter knows the conditions in both its own channel and the channel between itself and the primary receiver. Equipped with such knowledge, the secondary transmitter performs power control in order to achieve improved performance. Power control schemes also take into account the average interference constraints, and more effectively limit the interference inflicted on the primary users. The results obtained under such assumptions can be used to identify the performance gains and interference reductions 
achieved in the presence of channel knowledge when compared with those of $[8,9]$.

The rest of the article is organized as follows: In "Cognitive channel model and channel sensing" section, we discuss the channel model and analyze multi-channel sensing. We describe the channel state transition model in "State transition model" section under the assumption that the secondary users have perfect CSI and send the data at rates equal to the instantaneous channel capacity values. In "Interference power constraints" section, we analyze the received interference power at the primary receiver and apply this as a power constraint on the secondary users. In "Effective capacity" section, we define the effective capacity and find the optimal power distribution and show the criterion to choose the best channel. Numerical results are shown in "Numerical results" section, and conclusions are provided in "Conclusion" section.

\section{Cognitive channel model and channel sensing}

In this article, we consider a cognitive radio system in which secondary users sense $M$ channels. Since the transmission strategies of the cognitive radios depend on the activities of the primary users, it is desirable to have the cognitive radios sense multiple channels to improve the performance and more easily control the interference inflicted on the primary users. These available channels might be the white space television bands or the Industrial, Scientific and Medical (ISM) radio bands. In our model, we further assume that even if multiple channels are sensed, only one channel is always selected for transmission. Basically, we suppose that the cognitive radio employs narrowband transmission techniques.

We assume that channel sensing and data transmission are conducted in frames of duration $T$ seconds. In each frame, $N$ seconds is allocated for channel sensing while data transmission occurs in the remaining $T-N$ seconds. Transmission power and rate levels depend on the primary users' activities. If all of the channels are detected as busy, transmitter selects one channel with a certain criterion, and sets the transmission power and rate to $P_{k, 1}(i)$ and $r_{k, 1}(i)$, respectively, where $k \in\{1,2, \ldots, M\}$ is the index of the selected channel and $i=1,2, \ldots$ denotes the time index. Note that if $P_{k, 1}(i)=0$, transmitter stops sending information when it detects primary users in all channels. If at least one channel is sensed to be idle, data transmission is performed with power $P_{k, 2}(i)$ and at rate $r_{k, 2}(i)$. If multiple channels are detected as idle, then one idle channel is selected again considering a certain criterion.

The discrete-time channel input-output relation between the secondary transmitter and receiver in the $i$ th symbol duration in the $k$ th channel is given by

$$
y_{k}(i)=h_{k}(i) x_{k}(i)+n_{k}(i) \quad i=1,2, \ldots
$$

if the primary users are absent. On the other hand, if primary users are present in the channel, we have

$$
y_{k}(i)=h_{k}(i) x_{k}(i)+s_{k, p}(i)+n_{k}(i) \quad i=1,2, \ldots,
$$

where $x_{k}(i)$ and $y_{k}(i)$ denote the complex-valued channel input and output, respectively. In (1) and (2), $h_{k}(i)$ is the channel fading coefficient between the cognitive transmitter and the receiver. We assume that $h_{k}(i)$ has a finite variance, i.e., $\sigma_{h_{k}}^{2}<\infty$, but otherwise has an arbitrary distribution. We define $z_{k}(i)=\left|h_{k}(i)\right|^{2}$. We consider a block-fading channel model and assume that the fading coefficients stay constant for a block of duration $T$ seconds and change from one block to another independently in each channel. In (2), $s_{k, p}(i)$ represents the active primary user's faded signal arriving at the secondary receiver in the $k$ th channel, and has a variance $\sigma_{s_{k, p}}^{2}(i) . n_{k}(i)$ models the additive thermal noise at the receiver, and is a zero-mean, circularly symmetric, complex Gaussian random variable with variance $\mathbb{E}\left\{\left|n_{k}(i)\right|^{2}\right\}=\sigma_{n_{k}}^{2}$ for all $i$. We assume that the bandwidth of the $k$ channel is $B_{k}$.

In the absence of detailed information on primary users' transmission policies, energy-based detection methods are favorable for channel sensing. Knowing that wideband channels exhibit frequency selective features, we can divide the band into channels and estimate each received signal through its discrete Fourier transform [5]. The channel sensing can be formulated as a hypothesis testing problem between the noise $n_{k}(i)$ and the signal $s_{k, p}(i)$ in noise. Noting that there are $N B_{k}$ complex symbols in a duration of $N$ seconds in each channel with bandwidth $B_{k}$, the hypothesis test in channel $k$ can mathematically be expressed as follows

$$
\begin{aligned}
& \mathcal{H}_{k, 0}: y_{k}(i)=n_{k}(i), \quad i=1, \ldots, N B_{k} \\
& \mathcal{H}_{k, 1}: y_{k}(i)=s_{k, p}(i)+n_{k}(i), \quad i=1, \ldots, N B_{k} .
\end{aligned}
$$

For the above detection problem, the optimal NeymanPearson detector is given by [10]

$$
Y_{k}=\frac{1}{N B_{k}} \sum_{i=1}^{N B_{k}}\left|y_{k}(i)\right|^{2} \gtrless \mathcal{H}_{k, 0} \gamma_{k} .
$$

We assume that $s_{k, p}(i)$ has a circularly symmetric complex Gaussian distribution with zero-mean and variance $\sigma_{s_{k, p}}^{2}$. Assuming further that $\left\{s_{k, p}(i)\right\}$ are i.i.d., we can immediately conclude that the test statistic $Y_{k}$ is chisquare distributed with $2 N B_{k}$ degrees of freedom. In this case, the probabilities of false alarm and detection can be established as follows

$$
P_{k, f}=\operatorname{Pr}\left(Y_{k}>\gamma_{k} \mid \mathcal{H}_{k, 0}\right)=1-P\left(\frac{N B_{k} \gamma_{k}}{\sigma_{n_{k}}^{2}}, N B_{k}\right),
$$




$$
P_{k, d}=\operatorname{Pr}\left(Y_{k}>\gamma_{k} \mid \mathcal{H}_{k, 1}\right)=1-P\left(\frac{N B_{k} \gamma_{k}}{\sigma_{n_{k}}^{2}+\sigma_{s_{k, p}}^{2}}, N B_{k}\right),
$$

where $P(x, a)$ denotes the regularized lower gamma function and is defined as $P(x, a)=\frac{\gamma(x, a)}{\Gamma(a)}$ where $\gamma(x, a)$ is the lower incomplete gamma function and $\Gamma(a)$ is the Gamma function. In Figure 1, the probability of detection, $P_{d}$, and the probability of false alarm, $P_{f}$, are plotted as a function of the energy detection threshold, $\gamma$, for different values of channel detection duration. Note that the bandwidth is $B=10 \mathrm{kHz}$ and the block duration is $T=0.1 \mathrm{~s}$. We can see that when the detection threshold is low, $P_{d}$ and $P_{f}$ tend to be 1 , which means that the secondary user, always assuming the existence of an active primary user, transmits with power $P_{1}(i)$ and rate $r_{1}(i)$. On the other hand, when the detection threshold is high, $P_{d}$ and $P_{f}$ are close to zero, which means that the secondary user, being unable to detect the activity of the primary users, always transmits with power $P_{2}(i)$ and rate $r_{2}(i)$, possibly causing significant interference. The main purpose is to keep $P_{d}$ as close to 1 as possible and $P_{f}$ as close to 0 as possible. Therefore, we have to keep the detection threshold in a reasonable interval. Note that the duration of detection is also important since increasing the number of channel samples used for sensing improves the quality of channel detection.

In the hypothesis testing problem in (3), another approach is to consider $Y_{k}$ as Gaussian distributed, which is accurate if $N B_{k}$ is large [4]. In this case, the detection and false alarm probabilities can be expressed in terms of Gaussian Q-functions. We would like to note the rest of the analysis in the article does not depend on the specific expressions of the false alarm and detection probabilities. However, numerical results are obtained using (5) and (6).

\section{State transition model}

In this article, we assume that both the secondary receiver and transmitter have perfect channel side information, and hence perfectly know the realizations of the fading coefficients $\left\{h_{k}(i)\right\}$. We further assume that the wideband channel is divided into channels, each with bandwidth that is equal to the coherence bandwidth $B_{c}$. Therefore, we henceforth have $B_{k}=B_{c}$. With this assumption, we can suppose that independent flat fading is experienced in each channel. In order to further simplify the setting, we consider a symmetric model in which fading coefficients are identically distributed in different channels. Moreover, we assume that the background noise and primary users' signals are also identically distributed in different channels and hence their variances $\sigma_{n}^{2}$ and $\sigma_{s_{p}}^{2}$ do not depend on $k$, and the prior probabilities of each channel being occupied by the primary users are the same and equal to $\rho$. In channel sensing, the same energy threshold, $\gamma$, is applied in each channel. Finally, in this symmetric model, the transmission power and rate policies when the channels are idle or busy are the same for each channel. Due to the consideration of a symmetric model, we in the subsequent analysis drop the subscript $k$ in the expressions for the sake of brevity.

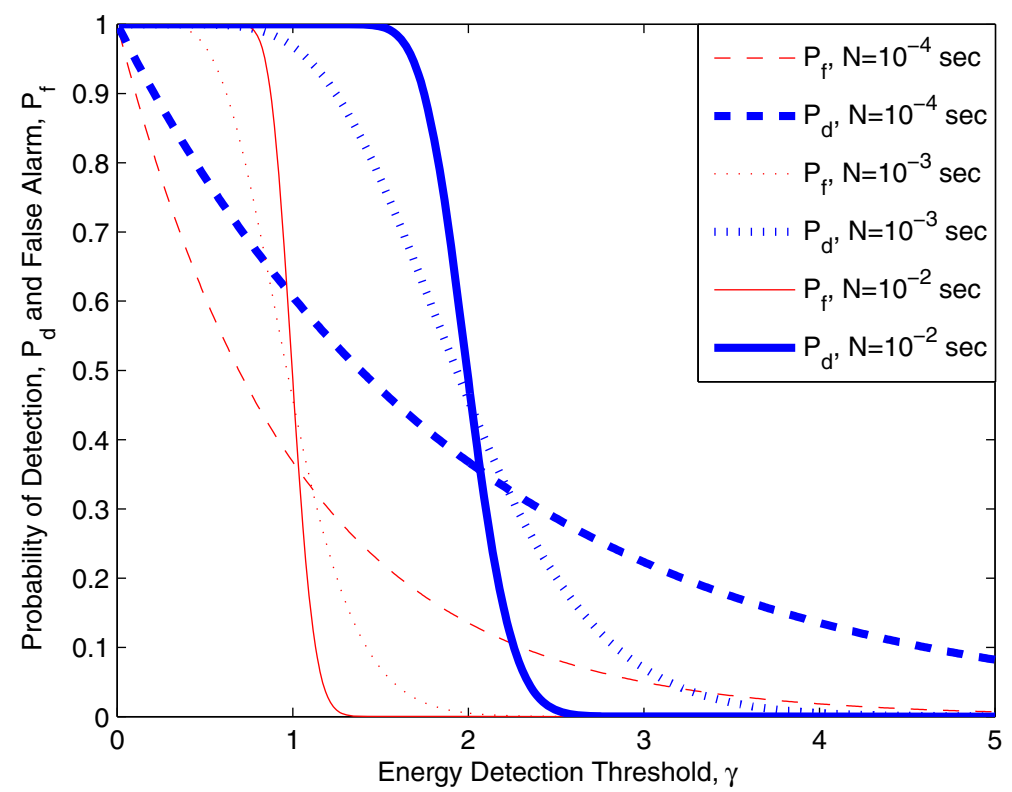

Figure 1 Probability of detection $\boldsymbol{P}_{\boldsymbol{d}}$ and false alarm $\boldsymbol{P}_{\boldsymbol{f}}$ versus energy detection threshold. 
First, note that we have the following four possible scenarios considering the correct detections and errors in channel sensing:

Scenario 1: All channels are detected as busy, and channel used for transmission is actually busy.

Scenario 2: All channels are detected as busy, and channel used for transmission is actually idle.

Scenario 3: At least one channel is detected as idle, and channel used for transmission is actually busy.

Scenario 4: At least one channel is detected as idle, and channel used for transmission is actually idle.

In each scenario, we have one state, namely either ON or OFF, depending on whether or not the instantaneous transmission rate exceeds the instantaneous channel capacity. Considering the interference $s_{p}(i)$ caused by the primary users as additional Gaussian noise, we can express the instantaneous channel capacities in the above four scenarios as follows:

Scenario 1: $C_{1}(i)=B_{c} \log _{2}\left(1+\operatorname{SNR}_{1}(i)\right)$.

Scenario 2: $C_{2}(i)=B_{c} \log _{2}\left(1+\mathrm{SNR}_{2}(i)\right)$.

Scenario 3: $C_{3}(i)=B_{c} \log _{2}\left(1+\mathrm{SNR}_{3}(i)\right)$.

Scenario 4: $C_{4}(i)=B_{c} \log _{2}\left(1+\mathrm{SNR}_{4}(i)\right)$.

Above, we have defined

$$
\begin{aligned}
& \operatorname{SNR}_{1}(i)=\frac{P_{1}(i) z(i)}{B_{c}\left(\sigma_{n}^{2}+\sigma_{s_{p}}^{2}\right)}, \operatorname{SNR}_{2}(i)=\frac{P_{1}(i) z(i)}{B_{c} \sigma_{n}^{2}} \\
& \operatorname{SNR}_{3}(i)=\frac{P_{2}(i) z(i)}{B_{c}\left(\sigma_{n}^{2}+\sigma_{s_{p}}^{2}\right)}, \operatorname{SNR}_{4}(i)=\frac{P_{2}(i) z(i)}{B_{c} \sigma_{n}^{2}}
\end{aligned}
$$

Note that $z(i)=|h(i)|^{2}$ denotes the fading power. In scenarios 1 and 2, the secondary transmitter detects all channels as busy and transmits the information at rate

$$
r_{1}(i)=B_{c} \log _{2}\left(1+\operatorname{SNR}_{1}(i)\right) .
$$

On the other hand, in scenarios 3 and 4, at least one channel is sensed as idle and the transmission rate is

$$
r_{2}(i)=B_{c} \log _{2}\left(1+\mathrm{SNR}_{4}(i)\right),
$$

since the transmitter, assuming the channel as idle, sets the power level to $P_{2}(i)$ and expects that no interference from the primary transmissions will be experienced at the secondary receiver (as seen by the absence of $\sigma_{s_{p}}^{2}$ in the denominator of $\mathrm{SNR}_{4}$ ).

In scenarios 1 and 2, transmission rate is less than or equal to the instantaneous channel capacity. Hence, reliable transmission at rate $r_{1}(i)$ is attained and channel is in the ON state. Similarly, the channel is in the ON state in scenario 4 in which the transmission rate is $r_{2}(i)$. On the other hand, in scenario 3 , transmission rate exceeds the instantaneous channel capacity (i.e., $r_{2}(i)>C_{3}(i)$ ) due to miss-detection. In this case, reliable communication cannot be established, and the channel is assumed to be in the OFF state. Note that the effective transmission rate in this state is zero, and therefore information needs to be retransmitted. We assume that this is accomplished through a simple ARQ mechanism.

For this cognitive transmission model, we initially construct a state transition model. While the ensuing discussion describes this model, Figure 2 provides a depiction. As seen in Figure 2, there are $(M+1)$ ON states and 1 OFF state. The single OFF state is the one experienced in scenario 3. The first ON state, which is the top leftmost state in Figure 2, is a combined version of the ON states in

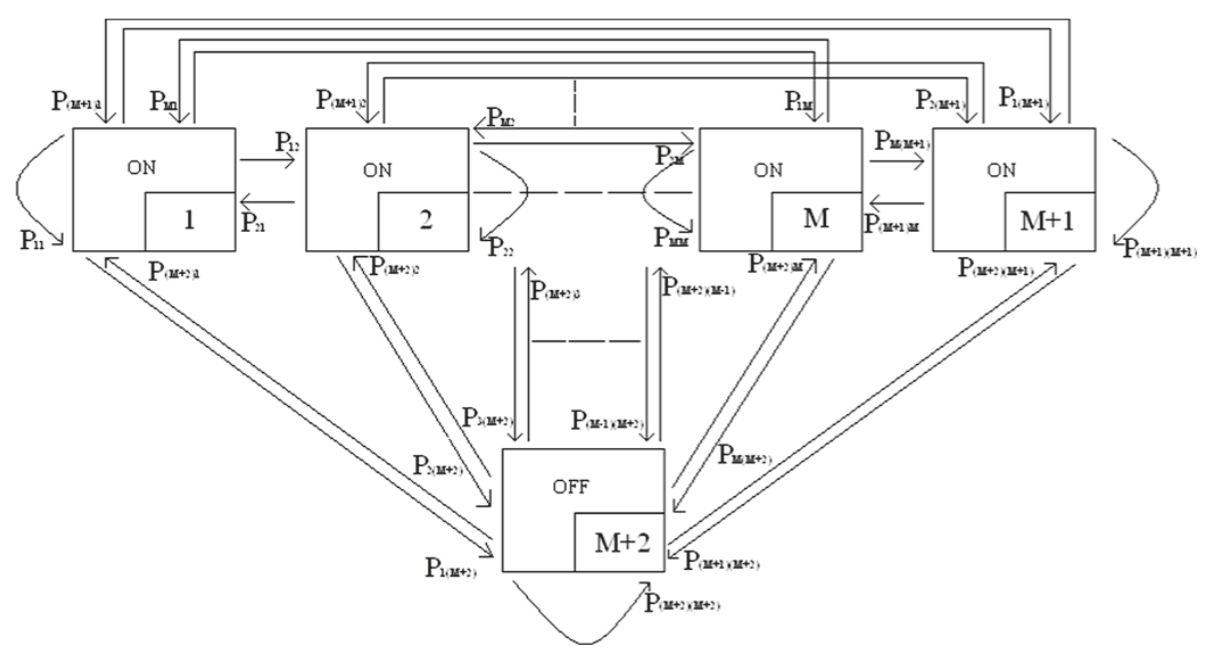

Figure 2 State transition model for the cognitive radio channel. The numbered label for each state is given on the lower-right corner of the box representing the state. 
scenarios 1 and 2 in both of which the transmission rate is $r_{1}(i)$ and the transmission power is $P_{1}(i)$. Note that all the channels are detected as busy in this first $\mathrm{ON}$ state. The remaining $\mathrm{ON}$ states labeled 2 through $(M+1)$ can be seen as the expansion of the $\mathrm{ON}$ state in scenario 4 in which at least one channel is detected as idle and the channel chosen for transmission is actually idle. More specifically, the $k$ th ON state for $k=2,3, \ldots,(M+1)$ is the ON state in which $(k-1)$ channels are detected as idle and the channel chosen for transmission is idle. Note that the transmission rate is $r_{2}(i)$ and the transmission power is $P_{2}(i)$ in all ON states labeled 2 through $(M+1)$.

Next, we characterize the state transition probabilities. State transitions occur every $T$ seconds. We can easily see that the probability of staying in the first ON state, in which all channels are detected as busy, is expressed as follows:

$$
p_{11}=\alpha^{M}
$$

where $\alpha=\rho P_{d}+(1-\rho) P_{f}$ is the probability that channel is detected as busy, and $P_{d}$ and $P_{f}$ are the probabilities of detection and false alarm, respectively, as defined in (6). Recall that $\rho$ denotes the probability that a channel is busy (i.e., there are active primary users in the channel). It is important to note that the transition probability in (10) is obtained under the assumptions that the primary user activity is independent among the channels and also from one block to another. Indeed, under the assumption of independence over the blocks, the state transition probabilities do not depend on the originating state ${ }^{a}$ and hence we have

$$
p_{11}=p_{21}=\cdots=p_{(M+1) 1}=p_{(M+2) 1}=\alpha^{M} \triangleq p_{1}
$$

where we have defined $p_{1}=p_{i 1}$ for all $i=1,2, \ldots, M+2$. Similarly, we can obtain for $k=2,3, \ldots, M+1$,
Now, we can easily observe that the transition probabilities for the OFF state are

$$
\begin{aligned}
p_{1(M+2)} & =p_{2(M+2)}=\cdots=p_{(M+1)(M+2)}=p_{(M+2)(M+2)} \\
& =1-\sum_{k=1}^{(M+1)} p_{1 k} \\
& =\sum_{k=1}^{M} \frac{M !}{(M-k) ! k !} \alpha^{(M-k)}(1-\alpha)^{k-1} \rho\left(1-P_{d}\right) \\
& \triangleq p_{(M+2) .}
\end{aligned}
$$

Then, we can easily see that the $(M+2) \times(M+2)$ state transition probability matrix can be expressed as

$$
\begin{aligned}
R & =\left[\begin{array}{rrcc}
p_{1,1} & \cdot & \cdot & p_{1,(M+2)} \\
\cdot & & & \cdot \\
\cdot & & & \cdot \\
p_{(M+2), 1} & \cdot & \cdot & p_{(M+2),(M+2)}
\end{array}\right] \\
& =\left[\begin{array}{cccc}
p_{1} & \cdot & \cdot & p_{(M+2)} \\
\cdot & & & \cdot \\
\cdot & & & \cdot \\
p_{1} & \cdot & \cdot & p_{(M+2)}
\end{array}\right]
\end{aligned}
$$

Note that $R$ has a rank of 1 . Note also that in each frame duration of $T$ seconds, $r_{1}(k)(T-N)$ bits are transmitted and received in state 1 , and $r_{2}(k)(T-N)$ bits are transmitted and received in states 2 through $(M+1)$, while the transmitted number of bits is assumed to be zero in state $(M+2)$.

\section{Interference power constraints}

In this section, we consider interference power constraints to limit the transmission powers of the secondary users and provide protection to primary users. In particular, we assume that the transmission power of the secondary users is constrained in such a way that the average interference power on the primary receiver is limited.

Note that interference to the primary users is caused in scenarios 1 and 3 . In scenario 1 , the channel is busy, and the secondary user, detecting the channel as busy,

$$
\begin{aligned}
& p_{1 k}=p_{2 k}=\cdots=p_{(M+1) k}=p_{(M+2) k}=P\left(\begin{array}{c}
(k-1) \text { out of } M \\
\text { channels are detected as idle }
\end{array} \text { and } \begin{array}{c}
\text { the channel chosen for transmission } \\
\text { is actually idle }
\end{array}\right) \\
& =\underbrace{\left(\begin{array}{c}
M \\
k-1
\end{array}\right) \alpha^{(M-k+1)}(1-\alpha)^{k-1}} \times \underbrace{\frac{(1-\rho)\left(1-P_{f}\right)}{1-\alpha}} \\
& \text { probability that }(k-1) \text { out of } M \text { channels probability that the channel chosen for } \\
& \text { are detected as idle transmission is actually idle } \\
& \text { given that it is detected as idle } \\
& =\frac{M !}{(M-k+1) !(k-1) !} \alpha^{(M-k+1)}(1-\alpha)^{k-2}(1-\rho)\left(1-P_{f}\right) \\
& \triangleq p_{k}
\end{aligned}
$$


transmits at power level $P_{1}$. Consequently, the instantaneous interference power experienced by the primary user is $P_{1} z_{s p}$ where $z_{s p}=\left|h_{s p}(i)\right|^{2}$ is the magnitude-square of the fading coefficient of the channel between the secondary transmitter and the primary user. Note also that the probability of being in scenario 1 (i.e., the probability of detecting all channels busy and having the chosen transmission channel as actually busy) is $\alpha^{(M-1)} \rho P_{d}$, as can easily be seen through an analysis similar to that in (13).

In scenario 3 , the secondary user, detecting the channel as idle, transmits at power $P_{2}$ although the channel is actually is busy. In this case, the instantaneous interference power is $P_{2} z_{s p}$. Since we consider power adaption, transmission power levels $P_{1}$ and $P_{2}$ in general vary with $z_{s p}$ and also with $z$, which is the power of the fading coefficient between the secondary transmitter and secondary receiver in the chosen transmission channel. Hence, in both scenarios, the instantaneous interference power levels depend on both $z_{s p}$ and $z$ whose distributions depend on the criterion with which the transmission channel is chosen and the number of available channels from which the selection is performed. For this reason, it is necessary in scenario 3 to separately consider the individual cases with different number of idle-detected channels. We have $M$ such cases. For instance, in the $k$ th case for $k=$ $1,2, \ldots, M$, we have $k$ channels detected as idle and the channel chosen out of these $k$ channels is actually busy. The probability of the $k$ th case can easily be found to be $\frac{M !}{(M-k) ! k !} \alpha^{(M-k)}(1-\alpha)^{k-1} \rho\left(1-P_{d}\right)$.

Following the above discussion, we can now express the average interference constraints as follows:

$$
\begin{aligned}
& \underbrace{\alpha^{(M-1)} \rho P_{d}}_{\begin{array}{c}
\text { probability of } \\
\text { scenario } 1
\end{array}} \underbrace{E\left\{P_{1} z_{s p}\right\}}_{\begin{array}{c}
\text { average interference } \\
\text { in scenario } 1
\end{array}} \\
& +\sum_{k=1}^{M} \underbrace{\frac{M !}{(M-k) ! k !}}_{\text {probability of the } k \text { th case of scenario } 3} \alpha^{M-k}(1-\alpha)^{k-1} \rho\left(1-P_{d}\right) \\
& \times \underbrace{E_{k}\left\{P_{2} z_{s p}\right\}}_{\begin{array}{c}
\text { average interference } \\
\text { in the } k \text { th case } \\
\text { of scenario 3 }
\end{array}} \leq I_{\text {avg }}
\end{aligned}
$$

Note from above that $I_{\text {avg }}$ is the constraint on the interference averaged over the distributions of $z$ and $z_{s p}$ (through the expectations), and also averaged over the probabilities of different scenarios and cases. It is important to note that the term $E_{k}\left\{P_{2} z_{s p}\right\}$, as discussed above, depends in general on the number of idle-detected channels, $k$. This dependence is indicated through the subscript $k$.
In a system with more strict requirements on the interference, the following individual interference constraints can be imposed

$$
\begin{aligned}
E\left\{P_{1} z_{s p}\right\} & \leq I_{0} \quad \text { and } \\
E_{k}\left\{P_{2} z_{s p}\right\} & \leq I_{k} \quad \text { for } \quad k=1,2, \ldots, M
\end{aligned}
$$

If, for instance, $I_{0}=I_{1}=I_{2}=\cdots=I_{M}$, then interference averaged over fading is limited by the same constraint regardless of which scenario is being realized.

In the subsequent parts of the article, we assume that an average interference power constraint in the form given in (18) is imposed.

\section{Effective capacity}

In this section, we identify the maximum throughput that the cognitive radio channel with the aforementioned state-transition model can sustain under interference power constraints and statistical QoS limitations imposed in the form of buffer or delay violation probabilities. ${ }^{\mathrm{b}} \mathrm{Wu}$ and Negi [11] defined the effective capacity as the maximum constant arrival rate that can be supported by a given channel service process while also satisfying a statistical QoS requirement specified by the QoS exponent $\theta$. If we define $Q$ as the stationary queue length, then $\theta$ is defined as the decay rate of the tail distribution of the queue length $Q$ :

$$
\lim _{q \rightarrow \infty} \frac{\log \operatorname{Pr}(Q \geq q)}{q}=-\theta
$$

Hence, we have the following approximation for the buffer violation probability for large $q_{\max }: \operatorname{Pr}\left(Q \geq q_{\max }\right) \approx$ $e^{-\theta q_{\max }}$. Therefore, larger $\theta$ corresponds to more strict QoS constraints, while the smaller $\theta$ implies looser constraints. In certain settings, constraints on the queue length can be linked to limitations on the delay and hence delay-QoS constraints. It is shown in [12] that $\operatorname{Pr}\{D \geq$ $\left.d_{\max }\right\} \leq c \sqrt{\operatorname{Pr}\left\{Q \geq q_{\max }\right\}}$ for constant arrival rates, where $D$ denotes the steady-state delay experienced in the buffer. In the above formulation, $c$ is a positive constant, $q_{\max }=$ $a d_{\max }$ and $a$ is the source arrival rate. Therefore, effective capacity provides the maximum arrival rate when the system is subject to statistical queue length or delay constraints in the forms of $\operatorname{Pr}\left(Q \geq q_{\max }\right) \leq e^{-\theta q_{\max }}$ or $\operatorname{Pr}\left\{D \geq d_{\max }\right\} \leq c e^{-\theta a d_{\max } / 2}$, respectively. Since the average arrival rate is equal to the average departure rate when the queue is in steady state [13], effective capacity can also be seen as the maximum throughput in the presence of such constraints. 
The effective capacity for a given QoS exponent $\theta$ is given by

$$
-\lim _{t \rightarrow \infty} \frac{1}{\theta t} \log _{e} E\left\{e^{-\theta S(t)}\right\}=-\frac{\Lambda(-\theta)}{\theta}
$$

where $S(t)=\sum_{k=1}^{t} r(k)$ is the time-accumulated service process, and $\{r(k), k=1,2, \ldots\}$ is defined as the discretetime, stationary, and ergodic stochastic service process. Note that $\Lambda(\theta)$ is the asymptotic log-moment generating function of $S(t)$, and is given by

$$
\Lambda(\theta)=\lim _{t \rightarrow \infty} \frac{1}{t} \log E\left[e^{\theta S(t)}\right] .
$$

The service rate according to the model described in "State transition model" section is $r(k)=r_{1}(k)(T-N)$ if the cognitive system is in state 1 at time $k$. Similarly, the service rate is $r(k)=r_{2}(k)(T-N)$ in the states between 2 and $(M+1)$. In the OFF state, instantaneous transmission rate exceeds the instantaneous channel capacity and reliable communication cannot be achieved. Therefore, the service rate in this state is effectively zero.

In the next result, we provide the effective capacity for the cognitive radio channel and state transition model described in the previous section. This result is obtained by directly making use of the characterization in ([14],Chap. 7, Example 7.2.7), where effective bandwidth of Markov modulated processes is formulated.

Theorem 1. For the cognitive radio channel with the state transition model given in "State transition model" section, the normalized effective capacity (in bits/s/ $\mathrm{Hz}$ ) under the average interference power constraint (18) is given by

$$
\begin{aligned}
& R_{E}(S N R, \theta)=-\frac{1}{\theta T B_{c}} \quad \max _{\alpha^{(M-1)} \rho P_{d} E\left\{P_{1} z_{s p}\right\}} \\
& +\sum_{k=1}^{M} \alpha^{M-k}(1-\alpha)^{k-1} \rho\left(1-P_{d}\right) \frac{M !}{(M-k) k ! k} E_{k}\left\{P_{2} z_{s p}\right\} \\
& \log _{e}\left(p_{1} E\left\{e^{-(T-N) \theta r_{1}}\right\}+\sum_{k=1}^{M} p_{k+1} E_{k}\left\{e^{-(T-N) \theta r_{2}}\right\}+p_{(M+2)}\right) .
\end{aligned}
$$

Above, $p_{k}$ for $k=1,2, \ldots, M+2$ denote the state transition probabilities defined in (11), (15), and (17). Note also that the maximization is with respect to the power adaptation policies $P_{1}$ and $P_{2}$.

Remark: In the effective capacity expression (23), the expectation $E\left\{P_{1} z_{s p}\right\}$ in the constraint and $E\left\{e^{-(T-N) \theta r_{1}}\right\}$ are with respect to the joint distribution of $\left(z, z_{s p}\right)$ of the channel selected for transmission when all channels are detected busy. The expectations $E_{k}\left\{P_{2} z_{s p}\right\}$ and $E_{k}\left\{e^{-(T-N) \theta r_{2}}\right\}$ are with respect to the joint distribution of $\left(z, z_{s p}\right)$ of the channel selected for transmission when $k$ channels are detected as idle.
Proof of Theorem 1: In ([14],Chap. 7, Example 7.2.7), it is shown for Markov modulated processes that

$$
\frac{\Lambda(\theta)}{\theta}=\frac{1}{\theta} \log _{e} \operatorname{sp}(\phi(\theta) R)
$$

where $\operatorname{sp}(\phi(\theta) R)$ is the spectral radius (i.e., the maximum of the absolute values of the eigenvalues) of the matrix $\phi(\theta) R, R$ is the transition matrix of the underlying Markov process, and $\phi(\theta)=\operatorname{diag}\left(\phi_{1}(\theta), \ldots, \phi_{(M+2)}(\theta)\right)$ is a diagonal matrix whose components are the moment generating functions of the processes in given states. The rates supported by the cognitive radio channel with the state transition model described in the previous section can be seen as a Markov modulated process and hence the setup considered in [14] can immediately be applied to our setting. Since the processes in the states are timevarying transmission rates, we can easily find that $\phi(\theta)=$ $\operatorname{diag}\left\{E\left\{e^{(T-N) \theta r_{1}}\right\}, E_{1}\left\{e^{(T-N) \theta r_{2}}\right\}, \ldots, E_{M}\left\{e^{(T-N) \theta r_{2}}\right\}, 1\right\}$. Then, we have

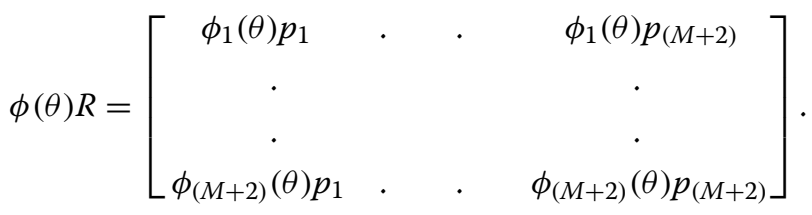

Since $\phi(\theta) R$ is a matrix with unit rank, we can readily find that

$$
\begin{aligned}
s p(\phi(\theta) R)= & \operatorname{trace}(\phi(\theta) R)=\phi_{1}(\theta) p_{1}+\phi_{2}(\theta) p_{2}+\cdots \\
& +\phi_{(M+1)}(\theta) p_{(M+1)}+\phi_{(M+2)}(\theta) p_{(M+2)} \\
= & p_{1} E\left\{e^{(T-N) \theta r_{1}}\right\}+p_{2} E_{1}\left\{e^{(T-N) \theta r_{2}}\right\} \\
& +\cdots+p_{(M+1)} E_{M}\left\{e^{(T-N) \theta r_{2}}\right\}+p_{(M+2)} .
\end{aligned}
$$

Then, combining (26) with (24) and (21), normalizing the expression with $T B_{c}$ in order to have the effective capacity in the units of bits/s/Hz, and considering the maximization over power adaptation policies, we reach to the effective capacity formula given in (23).

We note that one of the key steps in obtaining the effective capacity expression above is the observation that the matrix $R$ is of unit rank, which is due to independent state transitions, and consequently $\phi(\theta) R$ has only one nonzero eigenvalue. When the channel fading coefficients are assumed to be correlated, we will have a practically more appealing model. In such cases, state transition probabilities will depend on the originating states and the rank of the state-transition matrix $R$ will increase. At the same time, the effective capacity will still be formulated in terms of $\operatorname{sp}(\phi(\theta) R)$, the maximum of the absolute values of the eigenvalues of the matrix $\phi(\theta) R$. 
We would like to also note that the effective capacity expression in (23) is obtained for a given sensing duration $N$, detection threshold $\gamma$, and QoS exponent $\theta$. In the next section, we investigate the impact of these parameters on the effective capacity through numerical analysis. Before the numerical analysis, we first identify below the optimal power adaptation policies that the secondary users should employ.

Theorem 2. The optimal power adaptations for the secondary users under the constraint given in (18) are

$$
P_{1}= \begin{cases}\frac{\mu_{1}}{z}\left[\left(\frac{z}{z_{s p} \beta_{1} \lambda}\right)^{\frac{1}{c+1}}-1\right], & \frac{z}{z_{s p}} \geq \beta_{1} \lambda \\ 0, & \text { otherwise }\end{cases}
$$

and

$$
P_{2}= \begin{cases}\frac{\mu_{2}}{z}\left[\left(\frac{z}{z_{s p} \beta_{2} \lambda}\right)^{\frac{1}{c+1}}-1\right], & \frac{z}{z_{s p}} \geq \beta_{2} \lambda \\ 0, & \text { otherwise }\end{cases}
$$

where $\mu_{1}=B_{c}\left(\sigma_{n}^{2}+\sigma_{s_{p}}^{2}\right), \mu_{2}=\sigma_{n}^{2} B_{c}, c=B_{c}(T-$ $N) \theta / \log _{e} 2, \beta_{1}=\frac{\mu_{1} \rho P_{d}}{c \alpha}$ and $\beta_{2}=\frac{\rho\left(1-P_{d}\right) \mu_{2}}{c(1-\rho)\left(1-P_{f}\right)}$. $\lambda$ is a parameter whose value can be found numerically by satisfying the constraint (18) with equality.

Proof. Since logarithm is a monotonic function, the optimal power adaptation policies can also be obtained from the following minimization problem:

$$
\begin{aligned}
& \min \\
& \alpha^{(M-1)} \rho P_{d} E\left\{P_{1} z_{s p}\right\} \\
& \begin{array}{c}
+\sum_{k=1}^{M} \alpha^{M-k}(1-\alpha)^{k-1} \rho\left(1-P_{d}\right) \frac{M !}{(M-k) ! k !} E_{k}\left\{P_{2} z_{s p}\right\} \\
\leq I_{\text {avg }}
\end{array} \\
& p_{1} E\left\{e^{-(T-N) \theta r_{1}}\right\}+\sum_{k=1}^{M} p_{k+1} E_{k}\left\{e^{-(T-N) \theta r_{2}}\right\}
\end{aligned}
$$

It is clear that the objective function in (29) is strictly convex and the constraint function in (18) is linear with respect to $P_{1}$ and $P_{2}$. ${ }^{\mathrm{c}}$ Then, forming the Lagrangian function and setting the derivatives of the Lagrangian with respect to $P_{1}$ and $P_{2}$ equal to zero, we obtain

$$
\begin{aligned}
& {\left[\frac{\lambda \rho P_{d} z_{s p}}{\alpha}-\frac{c z}{\mu_{1}}\left(1+\frac{z P_{1}}{\mu_{1}}\right)^{-c-1}\right] \alpha^{M} f\left(z, z_{s p}\right)=0} \\
& {\left[\lambda \rho\left(1-P_{d}\right) z_{s p}-\frac{c(1-\rho)\left(1-P_{f}\right) z}{\mu_{2}}\left(1+\frac{z P_{2}}{\mu_{2}}\right)^{-c-1}\right]} \\
& \quad \times \sum_{k=1}^{M} \alpha^{M-k}(1-\alpha)^{k-1} \frac{M !}{(M-k) ! k !} f_{k}\left(z, z_{s p}\right)=0
\end{aligned}
$$

where $\lambda$ is the Lagrange multiplier. Above, $f\left(z, z_{s p}\right)$ denotes the joint distribution of $\left(z, z_{s p}\right)$ of the channel selected for transmission when all channels are detected busy. Hence, in this case, the transmission channel is chosen among $M$ channels. Similarly, $f_{k}\left(z, z_{s p}\right)$ denotes the joint distribution when $k$ channels are detected idle, and the transmission channel is selected out of these $k$ channels. Defining $\beta_{1}=$ $\frac{\mu_{1} \rho P_{d}}{c \alpha}$ and $\beta_{2}=\frac{\rho\left(1-P_{d}\right) \mu_{2}}{c(1-\rho)\left(1-P_{f}\right)}$, and solving (30) and (31), we obtain the optimal power policies given in (27) and (28).

Now, using the optimal transmission policies given in (27) and (28), we can express the effective capacity as follows

$$
\begin{aligned}
R_{E}(\mathrm{SNR}, \theta)= & -\frac{1}{\theta T B_{c}} \log _{e}\left(p_{1} E_{\beta_{1} \lambda}\left\{\left(\frac{z}{z_{s p} \beta_{1} \lambda}\right)^{-\frac{c}{c+1}}\right\}\right. \\
& \left.+\sum_{k=1}^{M} p_{k+1} E_{k, \beta_{2} \lambda}\left\{\left(\frac{z}{z_{s p} \beta_{2} \lambda}\right)^{-\frac{c}{c+1}}\right\}+p_{(M+2)}\right) .
\end{aligned}
$$

Above, the subscripts $\beta_{1} \lambda$ and $\beta_{2} \lambda$ in the expectations denote that the lower limits of the integrals are equal these values and not to zero. For instance, $E_{\beta_{1} \lambda}\left\{\left(\frac{z}{z_{s p} \beta_{1} \lambda}\right)^{-\frac{c}{c+1}}\right\}=\int_{\beta_{1} \lambda}^{\infty}\left(\frac{x}{\beta_{1} \lambda}\right)^{-\frac{c}{c+1}} f_{\frac{z}{z s p}}(x) d x$.

Until now, we have not specified the criterion with which the transmission channel is selected from a set of available channels. In (32), we can easily observe that the effective capacity depends only on the channel power ratio $\frac{z}{z_{s p}}$, and is increasing with increasing $\frac{z}{z_{s p}}$ due to the fact that the terms $\left(\frac{z}{z_{s p} \beta_{1} \lambda}\right)^{-\frac{c}{c+1}}$ and $\left(\frac{z}{z_{s p} \beta_{2} \lambda}\right)^{-\frac{c}{c+1}}$ are monotonically decreasing functions of $\frac{z}{z_{s p}}$. Therefore, the criterion for choosing the transmission band among multiple busy bands unless there is no idle band detected, or among multiple idle bands if there are idle bands detected should be based on this ratio of the channel gains. Clearly, the strategy that maximizes the effective capacity is to choose the channel (or equivalently the frequency band) with the highest ratio of $\frac{z}{z_{s p}}$. This is also intuitively appealing as we want to maximize $z$ to improve the secondary transmission and at the same time minimize $z_{s p}$ to diminish the interference caused to the primary users. Maximizing $\frac{z}{z_{s p}}$ provides us the right balance in the channel selection.

We define $x=\max _{i \in\{1,2, \ldots, M\}} \frac{z_{i}}{z_{s p, i}}$ where $\frac{z_{i}}{z_{s p, i}}$ is the ratio of the gains in the $i$ th channel. Assuming that these ratios are independent and identically distributed in different channels, we can express the pdf of $x$ as

$$
f_{x}(x)=M f_{\frac{z}{z_{s p}}}(x)\left[F_{\frac{z}{z_{s p}}}(x)\right]^{(M-1)},
$$

where $f_{\frac{z}{z_{s p}}}$ and $F_{\frac{z}{z_{s p}}}$ are the pdf and cumulative distribution function (cdf), respectively, of $\frac{z}{z_{s p}}$, the gain ratio in one channel. Now, the expectation $E_{\beta_{1} \lambda}\left\{\left(\frac{z}{z_{s p} \beta_{1} \lambda}\right)^{-\frac{c}{c+1}}\right\}$, 
which arises under the assumption that all channels are detected busy and the transmission channel is selected among these $M$ channels, can be evaluated with respect to the distribution in (33).

Similarly, we define $x_{k}=\max _{i \in\{1,2, \ldots, k\}} \frac{z_{i}}{z_{s p, i}}$ for $k=$ $1, \ldots, M$. The pdf of $x_{k}$ can be expressed as follows

$$
f_{x_{k}}(x)=k f_{\frac{z}{z_{s p}}}(x)\left[F_{\frac{z}{z_{s p}}}(x)\right]^{k-1} \quad k=1,2, \ldots, M .
$$

The expectation $E_{k, \beta_{2} \lambda}\left\{\left(\frac{z}{z_{s p} \beta_{2} \lambda}\right)^{-\frac{c}{c+1}}\right\}$ can be evaluated using the distribution in (34). Finally, after some calculations, we can write the effective capacity in integral form as

$$
\begin{aligned}
R_{E}(\mathrm{SNR}, \theta)= & -\frac{1}{\theta T B_{c}} \log _{e}\left\{M \alpha^{M} \int_{\beta_{1} \lambda}^{\infty} f_{\bar{z}}(x)\left[F_{\frac{z}{z s p}}(x)\right]^{(M-1)}\right. \\
& \times\left[\frac{\beta_{1} \lambda}{x}\right]^{\frac{c}{c+1}} d x(1-\rho)\left(1-P_{f}\right) M \\
& \times \int_{\beta_{2} \lambda}^{\infty} f_{\frac{z}{z s p}}(x)\left[\alpha+(1-\alpha) F_{\frac{z}{z s p}}(x)\right]^{(M-1)} \\
& \left.\times\left[\frac{\beta_{2} \lambda}{x}\right]^{\frac{c}{c+1}} d x+p_{(M+2)}\right\}
\end{aligned}
$$

\section{Numerical results}

In this section, we present numerical results for the effective capacity as a function of the channel sensing reliability (i.e., detection and false alarm probabilities) and the average interference constraints. Throughout the numerical results, we assume that QoS parameter is $\theta=0.1$, block duration is $T=1 \mathrm{~s}$, channel sensing duration is $N=0.1 \mathrm{~s}$, and the prior probability of each channel being busy is $\rho=0.1$

Before the numerical analysis, we first provide expressions for the probabilities of operating in each one of the four scenarios described in "State transition model" section. These probabilities are also important metrics in analyzing the performance. We have
In Figure 3, we plot these probabilities as a function of the detection probability $P_{d}$ for two cases in which the number of channels is $M=1$ and $M=10$, respectively. As expected, we observe that $P_{S_{1}}$ and $P_{S_{2}}$ decrease with increasing $M$. We also see that $P_{S_{3}}$ and $P_{S_{4}}$ are assuming small values when $P_{d}$ is very close to 1 . Note from Figure 1 that as $P_{d}$ approaches 1 , the false alarm probability $P_{f}$ increases as well.

\section{Rayleigh fading}

The analysis in the preceding sections apply for arbitrary joint distributions of $z$ and $z_{s p}$ under the mild assumption that the they have finite means (i.e., fading has finite average power). In this section, we consider a Rayleigh fading scenario in which the power gains $z$ and $z_{s p}$ are exponentially distributed. We assume that $z$ and $z_{s p}$ are mutually independent and each has unit-mean. Then, the pdf and cdf of $\frac{z}{z_{s p}}$ can be expressed as follows

$$
f_{\frac{z}{z s p}}(x)=\frac{1}{(x+1)^{2}} \quad x \geq 0 \text { and } F_{\frac{z}{z s p}}(x)=\frac{x}{x+1} \quad x \geq 0 .
$$

In Figure 4, we plot the effective capacity versus probability of detection, $P_{d}$, for different number of channels when the average interference power constraint normalized by the noise power is $\bar{I}_{\text {avg }}(d B)=10 \log _{10}\left(\frac{I_{\text {avg }}}{\sigma_{n_{p}}^{2} B_{c}}\right)=$ $0 \mathrm{~dB}$, where $\sigma_{n_{p}}^{2}$ is the noise variance at the primary user. We observe that with increasing $P_{d}$, the effective capacity is increasing due to the fact more reliable detection of the activity primary users leads to fewer miss-detections and hence the probability of scenario 3 or equivalently the probability of being in state $(M+2)$, in which the transmission rate is effectively zero, diminishes. We also interestingly see that the highest effective capacity is attained when $M=1$. Hence, secondary users seem to not benefit from the availability of multiple channels. This

$$
\begin{aligned}
& P\{\text { secondary system is in scenario } 1\}=P_{S_{1}}=\alpha^{(M-1)} \rho P_{d} \text {, } \\
& P\{\text { secondary system is in scenario } 2\}=P_{S_{2}}=\alpha^{(M-1)}(1-\rho) P_{f} \text {, } \\
& P\{\text { secondary system is in scenario } 3\}=P_{S_{3}}=\underbrace{\sum_{k=1}^{M}\left(\begin{array}{c}
M \\
k
\end{array}\right) \alpha^{M-k}(1-\alpha)^{k}}_{\text {probability that at least one channel }} \underbrace{\frac{\rho\left(1-P_{d}\right)}{1-\alpha}}_{\begin{array}{c}
\text { probability that the channel chosen } \\
\text { for transmission is actually busy }
\end{array}} \\
& \text { is detected as idle given that it is detected as idle } \\
& =\frac{\left(1-\alpha^{M}\right) \rho\left(1-P_{d}\right)}{1-\alpha}, \\
& P\{\text { secondary system is in scenario } 4\}=P_{S_{4}}=\frac{\left(1-\alpha^{M}\right)(1-\rho)\left(1-P_{f}\right)}{1-\alpha} \text {. }
\end{aligned}
$$




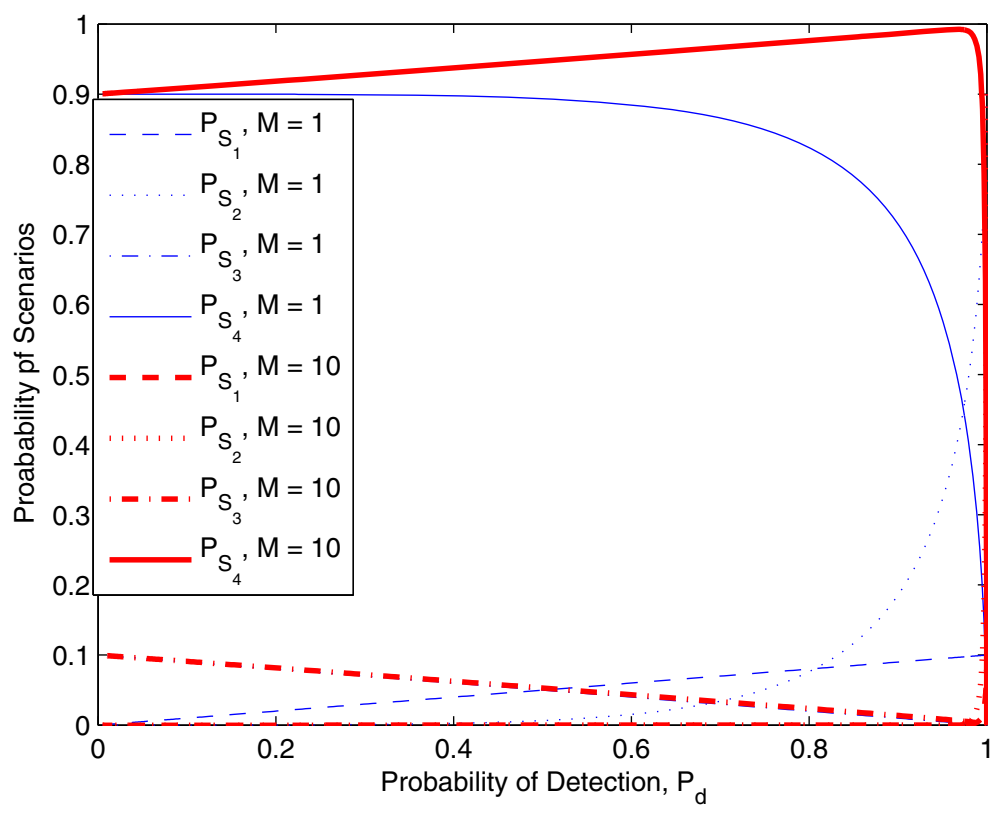

Figure 3 Probability of different scenarios versus probability of detection $P_{d}$ for different number of channels $M$.

is especially pronounced for high values of $P_{d}$. Although several factors and parameters are in play in determining the value of the effective capacity, one explanation for this observation is that the probabilities of scenarios 1 and 2, in which the secondary users transmit with power $P_{1}$, decrease with increasing $M$, while the probabilities of scenarios 3 and 4 increase as seen in (36). Note that in scenario 3, no reliable communication is possible and transmission rate is effectively zero. In Figure 5, we display similar results when $\bar{I}_{\text {avg }}=-10 \mathrm{~dB}$. Hence, secondary users operate under more stringent interference constraints. In this case, we note that $M=2$ gives the highest throughput while the performance with $M=1$ is strictly suboptimal.

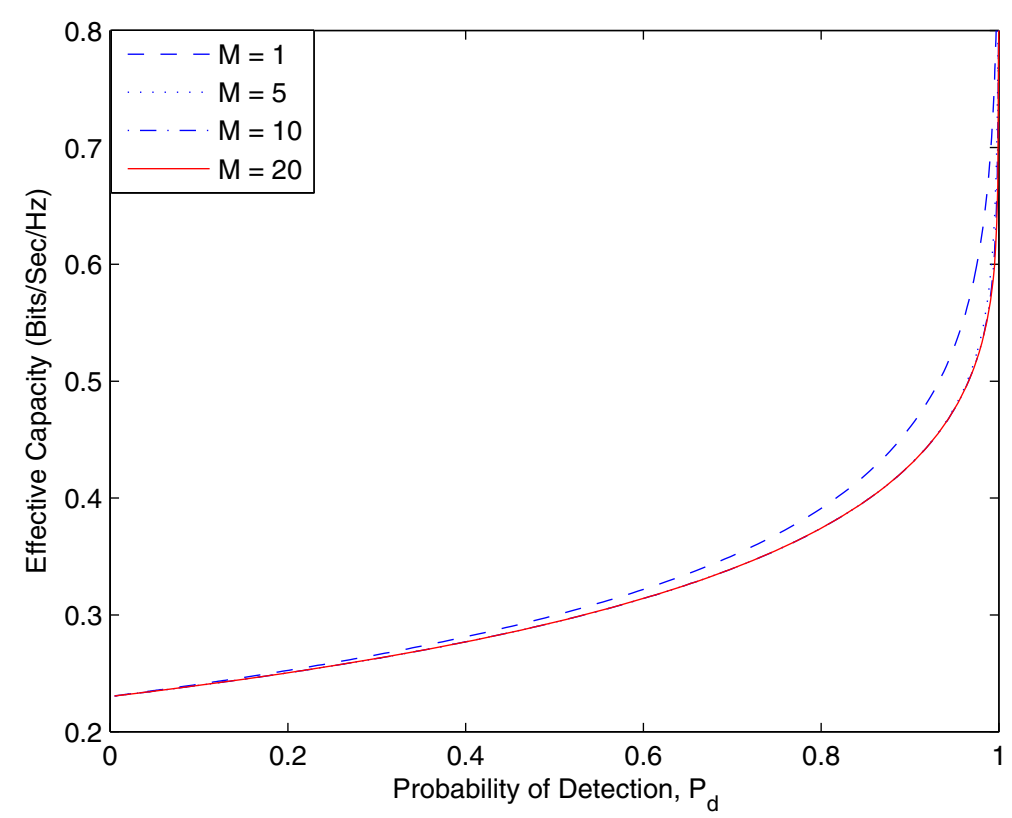

Figure 4 Effective capacity versus probability of detection $\boldsymbol{P}_{d}$ for different number of channels $M$ when $\bar{I}_{\mathrm{avg}}=\mathbf{0} \mathrm{dB}$. 


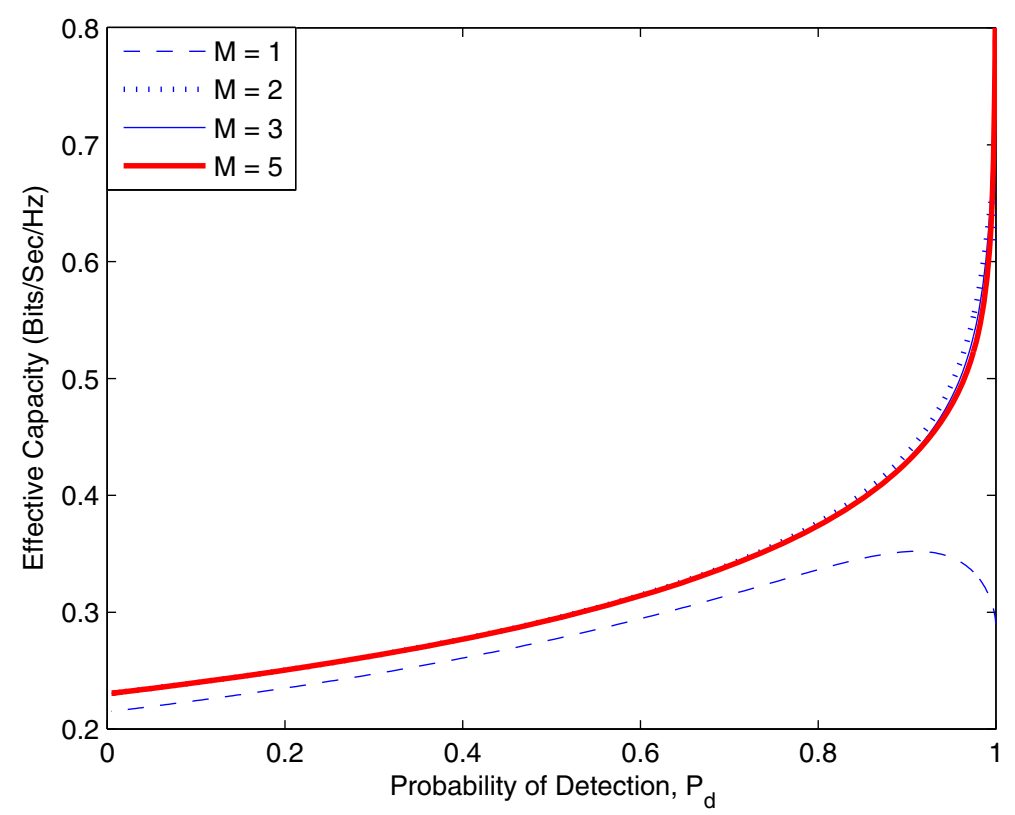

Figure 5 Effective capacity versus probability of detection $P_{d}$ for different number of channels $M$ when $\bar{I}_{\text {avg }}=-10 \mathrm{~dB}$.

In Figure 6, we show the effective capacities as a function $\bar{I}_{\text {avg }}(\mathrm{dB})$ for different values of $M$ when $P_{d}=0.9$ and $P_{f}=0.2$. Confirming our previous observation, we notice that as the interference constraint gets more strict and hence $\bar{I}_{\text {avg }}$ becomes smaller, a higher value of $M$ is needed to maximize the effective capacity. For instance, $M=10$ channels are needed when $\bar{I}_{\text {avg }}<-30 \mathrm{~dB}$. On the other hand, for approximately $\bar{I}_{\text {avg }}>-6 \mathrm{~dB}$, having $M=1$ gives the highest throughput.

Above, we have remarked that increasing the number of available channels from which the transmission channel is selected provides no benefit or can even degrade the performance of secondary users under certain conditions. On the other hand, it is important to note that increasing $M$

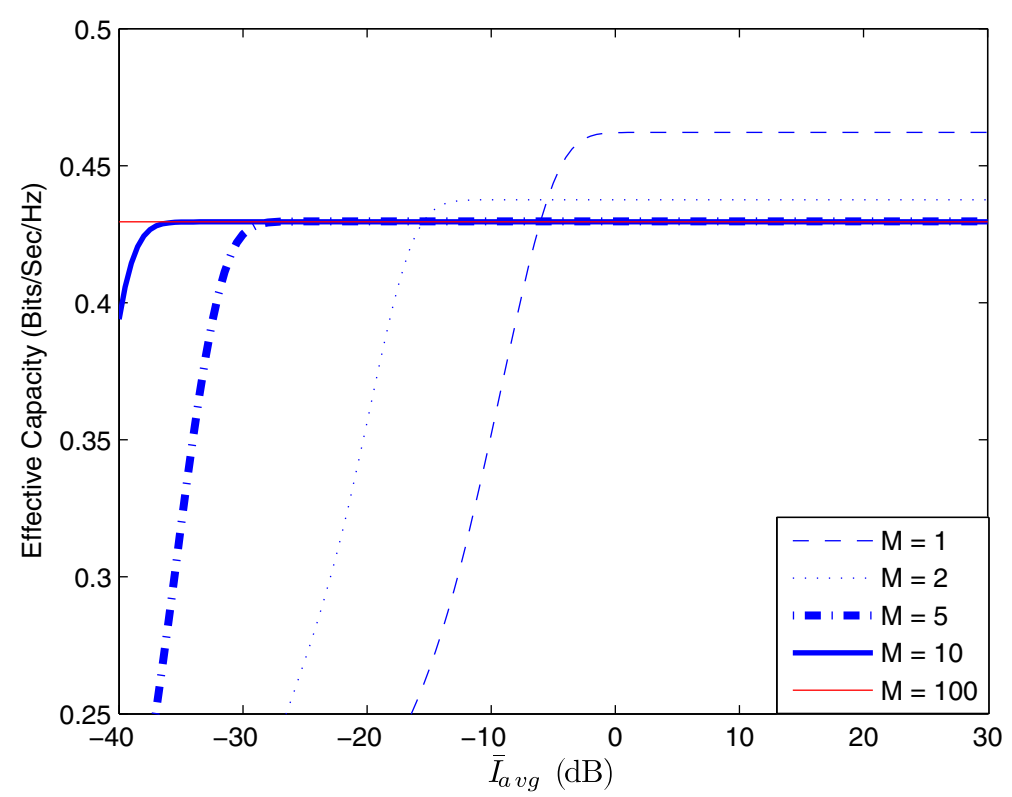

Figure 6 Effective capacity versus $\bar{I}_{\text {avg }}$ for different values of $M$ when $P_{d}=0.9$ and $P_{f}=0.2$ in the Rayleigh fading channel. 
always brings a benefit to the primary users in the form of decreased probability of interference. In order to quantify this type of gain, we consider below the probability that the channel selected for transmission is actually busy and hence the primary user in this channel experiences interference

$$
\begin{aligned}
P_{\text {int }}= & \operatorname{Pr}\left(\begin{array}{l}
\text { channel selected } \\
\text { for transmission } \\
\text { is actually busy }
\end{array}\right) \\
= & \operatorname{Pr}\left(\begin{array}{l}
\text { channel selected } \\
\text { for transmission and } \\
\text { is actually busy }
\end{array}\right. \\
& +\operatorname{Pr}\left(\begin{array}{l}
\text { channel selected channels are } \\
\text { for transmission as and } \\
\text { is actually busy }
\end{array}\right) \\
= & \left.P_{S_{1}}+P_{S_{3}} \begin{array}{c}
\text { at least one channel } \\
\text { is detected as idle }
\end{array}\right) \\
= & \rho \frac{1-\alpha^{M}-P_{d}+P_{d} \alpha^{(M-1)}}{1-\alpha} .
\end{aligned}
$$

Note that $P_{\text {int }}$ depends on $P_{d}$ and also $P_{f}$ through $\alpha=$ $\rho P_{d}+(1-\rho) P_{f}$. It can easily be seen that this interference probability $P_{\text {int }}$ decreases with increasing $M$ when $P_{d}>P_{f}$. As $M$ goes to infinity, we have $\lim _{M \rightarrow \infty} P_{\text {int }}=$ $\rho \frac{1-P_{d}}{1-\alpha}$. Indeed, in this asymptotic regime, $P_{\text {int }}$ becomes zero with perfect detection (i.e., with $P_{d}=1$ ). Note that secondary users transmit (if $P_{1}>0$ ) even when all channels are detected as busy. As $M \rightarrow \infty$, the probability of such an event vanishes. Also, having $P_{d}=1$ enables the secondary users to avoid scenario 3 . Hence, interference is not caused to the primary users.
In Figure 7, we plot $P_{\text {int }}$ vs. the detection probability for different values of $M$. We also display how the false alarm probability evolves as $P_{d}$ varies from 0 to 1 . It can be easily seen that while $P_{\text {int }}=\rho$ when $M=1$, a smaller $P_{\text {int }}$ is achieved for higher values of $M$ unless $P_{d}=1$. On the other hand, as also discussed above, we immediately note that $P_{\text {int }}$ monotonically decreases to 0 as $P_{d}$ increases to 1 when $M$ is unbounded (i.e., $M \rightarrow \infty$ ).

\section{Nakagami fading}

Nakagami fading occurs when multipath scattering with relatively large delay-time spreads occurs. Therefore, Nakagami distribution matches some empirical data better than many other distributions do. With this motivation, we also consider Nakagami fading in our numerical results. The pdf of the Nakagami- $m$ random variable $y=$ $|h|$ is given by $f_{y}(y)=\frac{2}{\Gamma(m)}\left(\frac{m}{2 \sigma_{y}^{2}}\right)^{m} y^{2 m-1} e^{-\frac{m y^{2}}{2 \sigma_{y}^{2}}}$ where $m$ is the number of degrees of freedom. If both $z_{s p}$ and $z$ have the same number of degrees of freedom, we can express the pdf of $x=\frac{z}{z_{s p}}$ as follows

$$
f_{x}(x)=\frac{\Gamma(2 m) x^{m-1}}{(x+1)^{2 m} \Gamma(m)^{2}} .
$$

Note also that Rayleigh fading is a special case of Nakagami fading when $m=1$. In our experiments, we
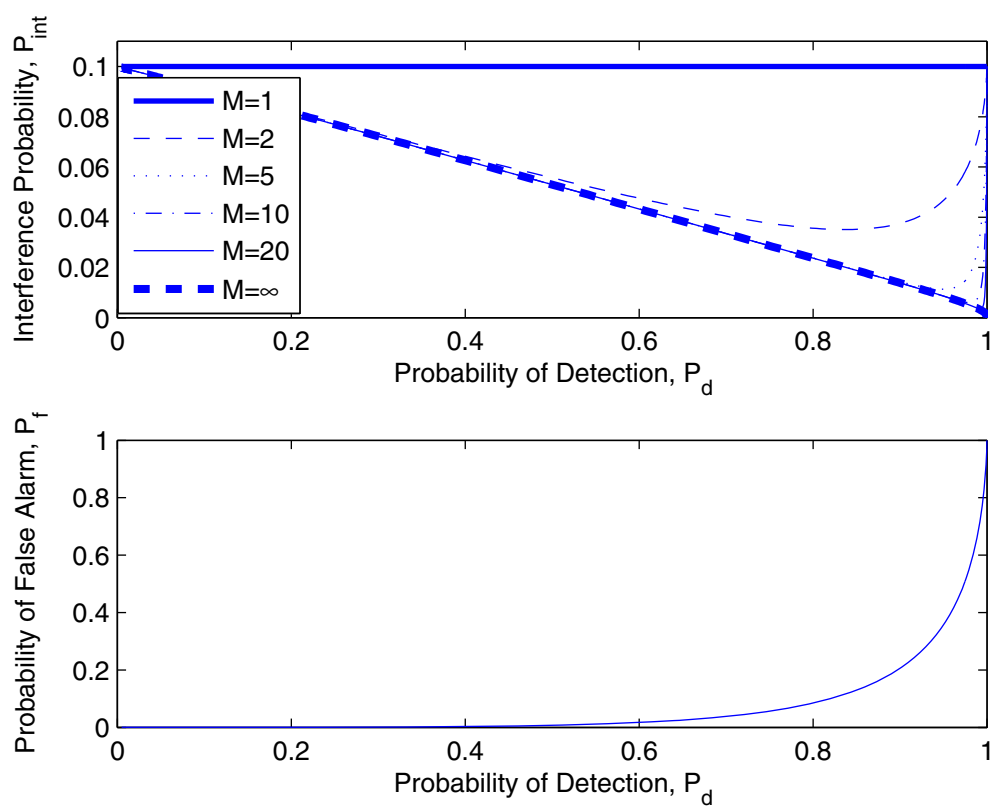

Figure $7 \boldsymbol{P}_{\text {int }}$ versus correct detection probability $\boldsymbol{P}_{\boldsymbol{d}}$ for different number of channels $\boldsymbol{M}$ in the upper figure. False alarm probability $P_{f}$ versus correct detection probability $P_{d}$ in the lower figure. 
consider the case in which $m=3$. Now, we can express the cdf of $x$ for $m=3$ as

$$
F_{x}(x)=1+\frac{15}{(x+1)^{4}}-\frac{10}{(x+1)^{3}}-\frac{6}{(x+1)^{4}} .
$$

In Figure 8 , we plot effective capacity versus $\bar{I}_{\text {avg }}(\mathrm{dB})$ for different values of $M$ when $P_{d}=0.9$ and $P_{f}=0.2$. Here, we again observe results similar to those in Figure 6. We obtain higher throughput by sensing more than one channel in the presence of strict interference constraints on cognitive radios.

\section{Conclusion}

In this article, we have studied the performance of cognitive transmission under QoS constraints and interference limitations. We have considered a scenario in which secondary users sense multiple channels and then select a single channel for transmission with rate and power that depend on both sensing decisions and fading. We have constructed a state transition model for this cognitive operation. We have meticulously identified possible scenarios and states in which the secondary users operate. These states depend on sensing decisions, true nature of the channels' being busy or idle, and transmission rates being smaller or greater than the instantaneous channel capacity values. We have formulated and imposed an average interference constraint on the secondary users. Under such interference constraints and also statistical QoS limitations in the form of buffer constraints, we have obtained the maximum throughput through the effective capacity formulation. Therefore, we have effectively analyzed the performance in a practically appealing setting in which both the primary and secondary users are provided with certain service guarantees. We have determined the optimal power adaptation strategies and the optimal channel selection criterion in the sense of maximizing the effective capacity. We have had several interesting observations through our numerical results. We have shown that improving the reliability of channel sensing expectedly increases the throughput. We have noted that sensing multiple channels is beneficial only under relatively strict interference constraints. At the same time, we have remarked that sensing multiple channels can decrease the chances of a primary user being interfered.

\section{Endnotes}

${ }^{a}$ Note that under the block-fading assumption, there is no memory in the state-transition model and hence the performance will depend on the steady-state probabilities of each state rather the transition probabilities.

${ }^{b}$ Note that interference constraints are imposed to provide a certain level of quality-of-service to the primary users, while buffer or delay constraints are used to statistically guarantee a quality-of-service level to the transmissions of the secondary users. Hence, the formulation in the paper effectively considers service guarantees for both the primary and secondary users. On the other hand, QoS constraints throughout the paper refer to buffer/delay constraints to avoid confusion.

${ }^{c}$ Strict convexity follows from the strict concavity of $r_{1}$ and $r_{2}$ in (8) and (9) with respect to $P_{1}$ and $P_{2}$ respectively,

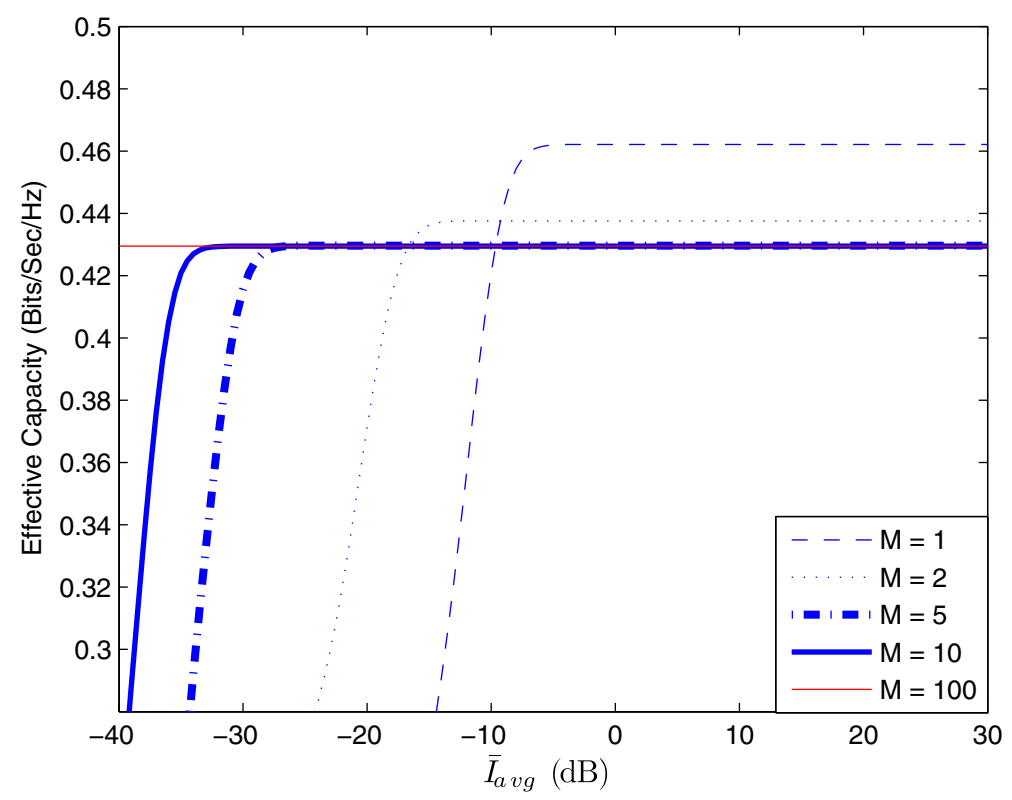

Figure 8 Effective capacity versus $\bar{I}_{\text {avg }}$ for different values of $M$ when $P_{d}=0.9$ and $P_{f}=0.2$ in the Nakagami- $m$ fading channel with $m=3$. 
strict convexity of the exponential function, and the fact that the nonnegative weighted sum of strictly convex functions is strictly convex ([15], Section 3.2.1).

\section{Competing interests}

The authors declare that they have no competing interests.

\section{Acknowledgements}

This study was supported by the National Science Foundation under Grants CNS-0834753 and CCF-0917265. The material in this article was presented in part at the IEEE International Conference on Communications (ICC), Cape Town, South Africa, May 2010.

\section{Author details}

${ }^{1}$ Institute of Communications Technology, Leibniz Universität Hannover, 30167 Hanover, Germany. ${ }^{2}$ Department of Electrical Engineering and Computer Science, Syracuse University, Syracuse, NY 13244, USA.

Received: 26 July 2011 Accepted: 5 September 2012

Published: 24 September 2012

\section{References}

1. V Asghari, S Aissa, in IEEE International Conference on Communications. Rate and power adaptation for increasing spectrum efficiency in cognitive radio networks, (Dresden, Germany, 14-18 June 2009), pp. 1-5

2. L Musavian, S Aissa, Capacity and power allocation for spectrum-sharing communications in fading channels. IEEE Trans. Wirel. Commun. 8(1), 148-156 (2009)

3. A Ghasemi, E Sousa, Spectrum sensing in cognitive radio networks: the cooperation-processing tradeoff. Wirel. Commun. Mobile Comput. 7(9), 1049-1060 (2007)

4. Y-C Liang, Y Zheng, ECY Peh, AT Hoang, Sensing-throughput tradeoff for cognitive radio networks. IEEE Trans. Wirel. Commun. 7(4), 1326-1337 (2008)

5. Z Quan, S Cui, AH Sayed, HV Poor, in IEEE International Conference on Communications. Wideband spectrum sensing in cognitive radio networks, (Beijing, China, 19-23 May 2008), pp. 901-906

6. L Musavian, S Aissa, in IEEE International Conference on Communications. Adaptive modulation in spectrum-sharing systems with delay constraints, (Dresden, Germany, 14-18 June 2009), pp. 1822-1826

7. L Musavian, S Aissa, in IEEE Global Communication Conference. Quality-of-service based power allocation in spectrum-sharing channels, (New Orleans, LA , USA, November 30-December 4, 2008), pp. 1-5

8. S Akin, MC Gursoy, Effective capacity analysis of cognitive radio channels for quality of service provisioning. IEEE Trans. Wirel. Commun. 9(11), 3354-3364 (2010)

9. S Akin, MC Gursoy, Performance analysis of cognitive radio systems under QoS constraints and channel uncertainty. IEEE Trans. Wirel. Commun. 10(9), 2883-2895 (2011)

10. HV Poor, An Introduction to Signal Detection and Estimation, 2nd edn. (Springer-Verlag, New York, 1994)

11. DWu, R Negi, Effective capacity: a wireless link model for support of quality of service. IEEE Trans. Wirel. Commun. 2(4), 630-643 (2003)

12. L Liu, J-F Chamberland, in IEEE International Symposium on Information Theory. On the effective capacities of multiple-antenna Gaussian channels, (Toronto, Ontario, Canada, 6-11 July 2008), pp. 2583-2587

13. C-S Chang, T Zajic, in IEEE Infocom. Effective bandwidths of departure processes from queues with time varying capacities, (Boston, MA, USA, 2-6 April 1995), vol. 3, pp. 1001-1009

14. C-S Chang, Performance Guarantees in Communication Networks. (Springer, New York, 1995)

15. S Boyd, L Vandenberghe, Convex Optimization. (Cambridge University Press, Cambridge, UK, 2004)

\section{Submit your manuscript to a SpringerOpen ${ }^{\circ}$ journal and benefit from:}

- Convenient online submission

- Rigorous peer review

- Immediate publication on acceptance

- Open access: articles freely available online

- High visibility within the field

- Retaining the copyright to your article

Submit your next manuscript at $\boldsymbol{\triangleright}$ springeropen.com 